\title{
SOME REMARKS ON THE STRUCTURE OF HOPF ALGEBRAS
}

\section{J. PETER MAY}

The purpose of this note is to give a new proof and generalization of Borel's theorem describing the algebra structure of connected commutative Hopf algebras and to discuss a certain functor $V$ that arises ubiquitously in algebraic topology. Using this functor, we shall construct another functor $W$ that accurately describes a large variety of not necessarily primitively generated Hopf algebras which occur in topology.

$K$ will denote a field of characteristic $p>0$. For any (nonnegatively) graded $K$-module $M$, we let $M^{+}=\{x \mid x \in M$, degree $x$ even $\}$ and $M^{-}=\{x \mid x \in M$, degree $x$ odd $\}$ if $p>2$; if $p=2$, we let $M^{+}=M$ and $M^{-}=0$. We recall that an Abelian restricted Lie algebra is a $K$-module $L$ together with a restriction ( $p$ th power operation) $\xi: L_{n} \rightarrow L_{p n}$, for $p n$ even, such that $\xi(k x)=k^{p} \xi(x)$ and $\xi(x+y)=\xi(x)+\xi(y)$ for $x, y \in L^{+}$ and $k \in K$. Define $V(L)=A(L) / I$, where $A(L)$ is the free commutative algebra generated by $L$ and $I$ is the ideal generated by $\left\{x^{p}-\xi(x) \mid x \in L^{+}\right\} . V(L)$ is a primitively generated commutative Hopf algebra with $P V(L)=L$. It has the universal property that if $i: L \rightarrow V(L)$ is the natural inclusion and if $f: L \rightarrow B$ is a morphism of Abelian restricted Lie algebras into a commutative algebra $B$ (with restriction the $p$ th power), then there exists a unique morphism of algebras $\tilde{f}: V(L) \rightarrow B$ such that $\tilde{f} i=f$. Of course, $V(L)$ is the universal (restricted) enveloping algebra of $L$ and is also defined for non-Abelian $L$, but it is the present case that is most frequently encountered in topology.

The following theorem is due to Milnor and Moore [7]. It can be derived from the Poincaré-Birkhoff-Witt theorem, and we shall see that it implies Borel's theorem quite directly.

THEOREM 1. If $A$ is a primitively generated Hopf algebra over $K$, then the natural map $V(P A) \rightarrow A$ is an isomorphism of Hopf algebras.

Now let $A$ be a connected commutative Hopf algebra over $K$. We study $A$ by relating it to its associated graded algebra $E^{0} A$. Thus filter $A$ by $F_{s} A=A$ if $s \geqq 0$ and $F_{s} A=(I A)^{-8}$ if $s<0$, where $I A$ is the augmentation ideal (positive degree elements) of $A$. Define $E_{s, t}^{0} A$ $=\left(F_{s} A / F_{s-1} A\right)_{s+t}$. Then $E^{0} A$ is a bigraded commutative Hopf algebra

Received by the editors April 3, 1969. 
with indecomposable elements $Q E^{0} A \cong E_{-1, *}^{0} A=Q A$. If $x \in E_{-1, *}^{0} A$, then $x$ is clearly primitive (since $E_{0, *}^{0} A=K$ and $E_{s, *}^{0} A=0$ for $s>0$ ). Therefore $E^{0} A$ is primitively generated, hence is isomorphic to $V\left(P E^{0} A\right)$. Now we have the following theorem, which is the analog in characteristic $p$ of Leray's theorem in characteristic zero. It seems not to have been previously noted. We remark that the theorem is valid even if $A$ is not coassociative.

TheOREM 2. Let $A$ be a connected commutative Hopf algebra over $K$. Let $f: Q A \rightarrow I A$ be any morphism of $K$-modules such that $\pi f=1$, $\pi: I A \rightarrow Q A$. Let $R A \subset A$ be the sub-Abelian restricted Lie algebra of $A$ generated by $f(Q A)$; thus $R A=f(Q A)+\left(\sum_{i \geqq 1} K f(Q A)^{p i}\right)$, where $K f(Q A)^{p i}$ is the sub $K$-module of $A$ generated by $\left\{x^{p i} \mid x \in f(Q A)^{+}\right\}$. Let $f: V(R A) \rightarrow A$ be the morphism of algebras induced from the inclusion $R A \subset A$ by the universal property of $V(R A)$. Then $\tilde{f}$ is an isomorphism of algebras.

Proof. Since $\tilde{f}$ is the identity on $f(Q A) \subset R A, Q \tilde{f}: Q V(R A) \rightarrow Q A$ is an epimorphism; since $A$ is connected, $\tilde{f}$ is therefore an epimorphism [7, Proposition 3.8]. Give $V(R A)$ and $A$ their product filtrations, as defined above. Since $\tilde{f}$ is a morphism of algebras, it is filtration preserving. These filtrations are complete $\left(A=\bigcup F_{s} A\right)$ since $A$ is connected, and it therefore suffices to prove that $E^{0} \tilde{f}$ is a monomorphism. Now $E^{0} \tilde{f}$ commutes with the coproduct on generators (elements of $\left.E_{-1, *}^{0}\right)$ since $E^{0} V(R)$ and $E^{0} A$ are primitively generated. Therefore $E^{0} \tilde{f}$ is a morphism of Hopf algebras and, by [7, Proposition 3.9], it suffices to prove that $E^{0} \tilde{f}$ is a monomorphism on primitive elements. Filter $R A$ by $F_{s} R A=R A \cap F_{s} V(R A)$. Then $E^{0} R A \subset E^{0} V(R A)$ and the induced map $V\left(E^{0} R A\right) \rightarrow E^{0} V(R A)$ is evidently an isomorphism of Hopf algebras. Since $\tilde{f}$ is the identity on $R A, \tilde{f}\left(F_{8} R A\right)=R A \cap F_{8} A$ and $E^{0} \tilde{f}$ restricted to $E^{0} R A$ is a monomorphism. Since $P E^{0} V(R A)$ $=E^{0} R A$, this completes the proof.

Thus to study the algebra structure of connected commutative Hopf algebras, it suffices to study Abelian restricted Lie algebras. The following theorem, together with the preceding one, will imply Borel's theorem.

THEOREM 3. Let $L$ be an Abelian restricted Lie algebra over a perfect field $K$, and suppose that $L$ is of finite type as a $K$-module. Then $L$ is isomorphic to a direct product of Abelian restricted Lie algebras on a single generator.

Proof. Clearly $L=L^{-} \times L^{+}$as an Abelian restricted Lie algebra. Since $L^{-}$is just a vector space, with no additional structure, we may 
as well assume $L=L^{+}$. Let $L(n)$ be the sub-Abelian restricted Lie algebra of $L$ generated by the $L_{i}$ for $i \leqq n . L=U_{n} L(n)$, and it suffices to prove the result for each $L(n)$. We proceed by induction on $n$. Now $L(0)=L_{0}$, and here the result is well known (see [2, pp. 43-44]). Thus assume the result for $L(n-1)$. We prove the result for $L(n)$ by induction on the number of generators of degree $n$ of $L(n)$ as an Abelian restricted Lie algebra, there being nothing to prove if there are no such generators. Let $L(n)$ have $q$ such generators and let $L^{\prime} \subset L(n)$ be the sub-Abelian restricted Lie algebra of $L(n)$ generated by $L(n-1)$ together with $q-1$ of these generators. The result holds for $L^{\prime}$ by the induction hypothesis, and $L^{\prime \prime}=L(n) / L^{\prime}$ is an Abelian restricted Lie algebra generated by one element, say $x$, of degree $n$. It suffices to prove that $L(n)$ is isomorphic to $L^{\prime} \times L^{\prime \prime}$. For this, we require a morphism $f: L^{\prime \prime} \rightarrow L(n)$ of Abelian restricted Lie algebras such that $\pi f=1$, where $\pi: L(n) \rightarrow L^{\prime \prime}$ is the projection. Define the height of an element of $L$ by $h(z)=i$ if $\xi^{i-1}(z) \neq 0$ but $\xi^{i}(z)=0$, and $h(z)=\infty$ if $\xi^{i}(z) \neq 0$ for all $i$. Of course, if $\pi(y)=x$, then $h(y) \geqq h(x)$ and, if $f$ exists and $f(x)=y$, then $h(y) \leqq h(x)$. Thus we can construct $f$ if and only if there exists $y \in L(n)$ such that $\pi(y)=x$ and $h(y)=h(x)$. If $h(x)=\infty$, then any $y$ such that $\pi(y)=x$ will do. Thus assume that $h(x)=r<\infty$. Since $K$ is perfect, $\xi^{r}\left(L^{\prime}\right)$ is a sub-Abelian restricted Lie algebra of $L^{\prime}$. Let $M^{\prime}=L^{\prime} / \xi^{r}\left(L^{\prime}\right)$ and $M=L / \xi^{r}\left(L^{\prime}\right)$. Let $\sigma: L \rightarrow M$ and $\rho: M \rightarrow M / M^{\prime}$ be the projections. We may identify $M / M^{\prime}$ with $L^{\prime \prime}$ and then $\pi=\rho \sigma$. Choose $z \in M$ and $w \in L$ such that $\rho(z)=x$ and $\sigma(w)=z . M_{q}^{\prime}=0$ for $q \geqq p^{r} n$ by construction and $L_{q}^{\prime \prime}=0$ for $q \geqq p^{r} n$ by the definition of $r$. Therefore $M_{q}=0$ for $q \geqq p^{r} n$ and $\xi^{r}(z)=0$. Thus $\sigma\left(\xi^{r}(w)\right)=0$ and $\xi^{r}(w) \in \xi^{r}\left(L^{\prime}\right)$, say $\xi^{r}\left(w^{\prime}\right)=\xi^{r}(w)$, w $w^{\prime} \in L^{\prime}$. Let $y=w$ $-w^{\prime}$. Then $\pi(y)=\rho \sigma(w)=x$ and $\xi^{r}(y)=0$, and $f$ can indeed be constructed.

The following two structure theorems for commutative Hopf algebras result by combining the previous theorems; the first generalizes a result of Milnor and Moore [7, Theorem 7.16] and the second is Borel's Theorem [1, Theorem 6.1].

THEOREM 4. If $A$ is a primitively generated commutative Hopf algebra (not necessarily connected) of finite type over a perfect field $K$, then $A$ is isomorphic as a Hopf algebra to a tensor product of Hopf algebras on a single generator.

Proof. $A \cong V(P A)$ by Theorem 1. $P A$ is isomorphic to a direct product $X_{i} L_{i}$ of monogenic Abelian restricted Lie algebras by Theorem 3. $V\left(\times_{i} L_{i}\right)=\otimes_{i} V\left(L_{i}\right)$ as a Hopf algebra by [7, Proposition 6.5], and each $V\left(L_{i}\right)$ is clearly monogenic. 
We observe that if $L$ is monogenic, then $V(L)=\oplus E^{0} V(L)$ as a Hopf algebra, where $\oplus E^{0} V(L)$ is $E^{0} V(L)$ regraded by total degree. Since the functor $E^{0}$ commutes with tensor products, we have, under the hypotheses of the theorem, that $V(P A)$ is isomorphic to $\oplus E^{0} V(P A)$ as a Hopf algebra. This proves the following corollary.

Corollary 5. Under the hypotheses of Theorem 4, $A$ is isomorphic as a Hopf algebra to $\oplus E^{0} \mathrm{~A}$.

Theorem 6. If $A$ is a connected commutative Hopf algebra of finite type over a perfect field $K$, then $A$ is isomorphic as an algebra to a tensor product of Hopf algebras on a single generator.

Proof. $A=V(R A)$ by Theorem 2 and the result follows from Theorem 3 by the argument used to prove Theorem 4 .

CoRollary 7. Under the hypotheses of Theorem $6, A$ is isomorphic as an algebra to $\oplus E^{0} A$.

REMARK 8. With the notion of Abelian restricted Lie algebra replaced by that of Abelian Lie algebra (i.e., vector space) and with $V(L)$ replaced by $U(L)=A(L)$, precisely the same arguments go through for fields of characteristic zero; here Theorem 3 is a triviality.

Theorem 2 suggests that $V$ should be regarded as a functor from Abelian restricted Lie algebras to commutative algebras; it is then adjoint to the obvious forgetful functor. The following two examples are instances of the occurrence of this functor in algebraic topology.

EXAmple 9. Let $L$ be an unstable module over the $\bmod p$ Steenrod algebra $A$ [8]. Define $\xi$ on $L$ by $\xi(x)=P^{i}(x)$ if $2 i=\operatorname{deg} x$ and $p>2$ or by $\xi(x)=\operatorname{Sq}^{i}(x)$ if $i=\operatorname{deg} x$ and $p=2$. Then the algebra $V(L)$ is an $A$-algebra, the free unstable $A$-algebra generated by $L$.

EXAMPLE 10. Let $L$ be an allowable module over the mod $p$ DyerLashof algebra $R$ [4], [5]. Define $\xi$ on $L$ by $\xi(x)=Q^{i}(x)$ if $2 i=\operatorname{deg} x$ and $p>2$ or by $\xi(x)=Q^{i}(x)$ if $i=\operatorname{deg} x$ and $p=2$. Then the algebra $V(L)$ is an $R$-algebra, the free allowable $R$-algebra generated by $L$.

In the applications, $L$ is of ten the cokernel $J C$ of the unit $\eta: K \rightarrow C$ of a coalgebra $C$, and $C$ determines a structure of Hopf algebra, not always primitively generated, on $V(J C)$. We complete this paper by studying this situation algebraically and giving a few examples.

Definition 11. A restricted coalgebra is a graded $K$-module $C$ which is both a unital coalgebra and an Abelian restricted Lie algebra such that $\eta: K \rightarrow C$ and $\epsilon: C \rightarrow K$ are morphisms of coalgebras and of Abelian restricted Lie algebras (where $\xi(k)=k^{p}$ on $K$ ) and such that $\psi \xi=(\xi \otimes \xi) \psi$ on $C^{+}$. Define the universal enveloping Hopf algebra of 
$C$ to be a commutative Hopf algebra $W(C)$ together with a morphism of restricted coalgebras $i: C \rightarrow W(C)$ such that for any morphism of restricted coalgebras $f: C \rightarrow B$ into a commutative Hopf algebra $B$, there exists a unique morphism of Hopf algebras $\bar{f}: W(C) \rightarrow B$ such that $\tilde{f} i=f$. Clearly $W(C)$ is unique, up to natural isomorphism, if it exists.

Proposition 12. Let $C$ be a restricted coalgebra. Then $V(J C)$ admits a structure of Hopf algebra such that $i: C \rightarrow V(J C)$ is a morphism of restricted coalgebras, and $V(J C)$ is the universal enveloping Hopf algebra $W(C)$ of $C$.

Proof. Recall that $V(J C)=A(J C) / I$, where $I$ is the ideal generated by $\left\{x^{p}-\xi(x) \mid x \in(J C)^{+}\right\}$. Since $A(J C)$ is free commutative, $\psi: C \rightarrow C \otimes C \subset A(J C) \otimes A(J C)$ induces a morphism of algebras $\psi: A(J C) \rightarrow A(J C) \otimes A(J C)$. This gives $A(J C)$ a structure of Hopf algebra. To show that $V(J C)$ is a Hopf algebra, we must verify that $I$ is a Hopf ideal, $\psi(I) \subset A(J C) \otimes I+I \otimes A(J C)$. Let $x \in(J C)^{+}$and write $\psi(x)=\sum x^{\prime} \otimes x^{\prime \prime}$. Then we have the relation:

$$
\begin{aligned}
\psi\left(x^{p}-\xi(x)\right) & =\psi(x)^{p}-\psi \xi(x)=\sum\left(x^{\prime}\right)^{p} \otimes\left(x^{\prime \prime}\right)^{p}-\sum \xi\left(x^{\prime}\right) \otimes \xi\left(x^{\prime \prime}\right) \\
& =\sum\left(\left(x^{\prime}\right)^{p}-\xi(x)\right) \otimes\left(x^{\prime \prime}\right)^{p}+\sum \xi\left(x^{\prime}\right) \otimes\left(\left(x^{\prime \prime}\right)^{p}-\xi\left(x^{\prime \prime}\right)\right),
\end{aligned}
$$

and this proves our claim. Let $f: C \rightarrow B$ be a morphism of restricted coalgebras, where $B$ is a commutative Hopf algebra. By the universal property of $V(J C)$, there exists a unique morphism of algebras $\tilde{f}: V(J C) \rightarrow B$ such that $\tilde{f} i=f$. Since $i(J C)$ generates $V(J C)$ as an algebra and $\bar{f}$ commutes with the coproduct on the image of $i, \tilde{f}$ is indeed a morphism of Hopf algebras.

$W$ is a functor from restricted coalgebras to commutative Hopf algebras; it is clearly adjoint to the obvious forgetful functor. Of course, if $C$ is totally primitive (every element of $J C$ is primitive) then $W(C)$ is primitively generated.

Example 13. In Example 10, let $L=J C$ where $C$ is a unital $A$-coalgebra. The identity $\psi \xi=(\xi \otimes \xi) \psi$ follows from the Cartan formula and $W(C)$ is a Hopf algebra over $A . H^{*}\left(K\left(Z_{p}, n\right) ; Z_{p}\right)$ has the form $W(C)$ for a certain totally primitive $C . H^{*}\left(\operatorname{Spin}(n) ; Z_{2}\right)$, for most values of $n$, has the form $W(C)$ where $C$ is not totally primitive [6].

Example 14. In Example 11, let $L=J C$ where $C$ is a unital $R$ coalgebra. The identity $\psi \xi=(\xi \otimes \xi) \psi$ follows from the Cartan formula and $W(C)$ is a Hopf algebra over $R$. If $Q X=\lim \Omega^{n} S^{n} X$ and $X$ is connected, then $H_{*}(Q X)=W(C)$ as a Hopf algebra where $C$ is a certain $R$-coalgebra determined by $H_{*}(X)[5]$. 
Example 15. Let $A_{*}$ denote the dual of the $\bmod p$ Steenrod algebra. $A_{*}=E\left\{\tau_{i} \mid i \geqq 0\right\} \otimes P\left\{\xi_{j} \mid j \geqq 1\right\}$ as an algebra and, with $\xi_{0}=1$,

$$
\psi\left(\tau_{i}\right)=\tau_{i} \otimes \xi_{0}+\sum_{k=0}^{i} \xi_{i-k}^{p k} \otimes \tau_{k} \quad \text { and } \quad \psi\left(\xi_{j}\right)=\sum_{k=0}^{j} \xi_{j-k}^{p k} \otimes \xi_{k} .
$$

Let $C$ be the sub-restricted coalgebra of $A_{*}$ spanned by all $\tau_{i}$ and $\xi_{j}^{p k}$ (with restriction the $p$ th power operation). Then $A_{*}=W(C)$ as a Hopf algebra. If $\left(E^{0} A\right)_{*}$ is the dual of the associated graded algebra of $A$, then $\left(E^{0} A\right)_{*}=W\left(E^{0} C\right)$ as a Hopf algebra, where $E^{0} C$ is the restricted coalgebra which is isomorphic to $C$ as a coalgebra but has its $p$ th power operation identically zero [3]. The same results are valid for $p=2$, with all mention of the $\tau_{i}$ suppressed.

In connection with Examples 13 and 14, it would be of interest to have an intrinsic algebraic characterization of those connected commutative Hopf algebras $A$ which have the form $W(C)$ for some $C$. By Theorem 2, a necessary and sufficient condition is the existence of $f: Q A \rightarrow I A, \pi f=1$, such that $R A \oplus K$ is a sub-coalgebra (hence a sub-restricted coalgebra) of $A$.

\section{BIBLIOGRAPHY}

1. A. Borel, Sur la cohomologie des espaces fibres principaux et des espaces homogènes de groupes de Lie compacts, Ann. of Math. (2) 57 (1953), 115-207.

2. N. Jacobson, The theory of rings, Math. Surveys, vol. 1, Amer. Math. Soc., Providence, R. I., 1943.

3. J. P. May, The cohomology of the Steenrod algebra, Ph.D. Thesis, Princeton University, Princeton, N. J., 1964.

4. - The Dyer-Lashof algebra and infinite loop spaces (to appear).

5. —- Allowable R-modules and $H_{*}(Q X)$ (to appear).

6. The cohomology of $B \operatorname{Spin}(n)$ (to appear).

7. J. Milnor and J. C. Moore, On the structure of Hopf algebras, Ann. of Math (2) 81 (1965), 211-264.

8. N. E. Steenrod and D. B. A. Epstein, Cohomology operations, Ann. of Math. Studies, No. 50, Princeton Univ. Press, Princeton, N. J., 1962.

University of Chicago 